\title{
A three-constituent damage model for arterial clamping in computer-assisted surgery
}

\author{
Nele Famaey · Jos Vander Sloten · Ellen Kuhl
}

Received: 18 October 2011 / Accepted: 24 February 2012 (c) Springer-Verlag 2012

\begin{abstract}
Robotic surgery is an attractive, minimally invasive and high precision alternative to conventional surgical procedures. However, it lacks the natural touch and force feedback that allows the surgeon to control safe tissue manipulation. This is an important problem in standard surgical procedures such as clamping, which might induce severe tissue damage. In complex, heterogeneous, large deformation scenarios, the limits of the safe loading regime beyond which tissue damage occurs are unknown. Here, we show that a continuum damage model for arteries, implemented in a finite element setting, can help to predict arterial stiffness degradation and to identify critical loading regimes. The model consists of the main mechanical constituents of arterial tissue: extracellular matrix, collagen fibres and smooth muscle cells. All constituents are allowed to degrade independently in response to mechanical overload. To demonstrate the modularity and portability of the proposed model, we implement it in a commercial finite element programme, which allows to keep track of damage progression via internal variables. The loading history during arterial clamping is simulated through four successive steps, incorporating residual strains. The results of our first prototype simulation demonstrate significant regional variations in smooth muscle cell damage. In three additional steps, this damage is evaluated by simulating an isometric contraction experiment. The entire finite element simulation is finally compared with actual in vivo experiments. In the short term, our computational simulation tool can be useful to optimise surgical tools with the goal to minimise tissue damage. In the long term, it can potentially be used to inform computer-assisted surgery and identify safe
\end{abstract}

N. Famaey $(\varangle) \cdot$ J. V. Sloten $\cdot$ E. Kuhl Celestijnenlaan 300C, 3001 Leuven, Belgium

e-mail: nele.famaey@mech.kuleuven.be loading regimes, in real time, to minimise tissue damage during robotic tissue manipulation.

Keywords Artery - Damage - Smooth muscle cells . Active contraction · Residual stress $\cdot$ Finite elements

\section{Introduction}

For the past two decades, computer-assisted surgery has revolutionised surgical treatment in various different fields. Initially developed to surgically manipulate the brain, see Kwoh et al. (1988), robotic surgery has now gained widespread use. The da Vinci surgical system, for example, offers a computer-enhanced surgical option for complex cardiovascular procedures, see Mohr et al. (2001). Robotic surgery enables minimally invasive and high-precision treatment. However, in contrast to conventional surgeries, robotic surgery inherently lacks the natural touch and force feedback. This is an important problem during common surgical procedures such as grasping, cutting, stapling, clipping and clamping, which may induce severe tissue damage when not controlled appropriately.

To illustrate these effects, within this manuscript, we focus in particular on arterial clamping, which always entails a certain degree of undesired iatrogenic tissue damage (Barone et al. 1989). Research has been directed towards decreasing this unnecessary intra-operative trauma, for example through the design of less traumatic surgical instruments (Gupta et al. 1997). Obviously, the effectiveness of these new designs and techniques depends on how well damage mechanisms are understood and how accurately thresholds for safe tissue loading can be defined.

An important aspect is the accurate modelling of the loading and the resulting damage process. This article describes 
a new material model for cardiovascular tissue, which is an extension of the Holzapfel-material model for arterial tissue (Holzapfel et al. 2000), incorporating smooth muscle cell activation according to Murtada et al. (2010) and damage according to Balzani et al. (2006). The model is suitable to simulate the damage process during the clamping of an artery. It displays the decrease of active force generation in smooth muscle cells due to the sustained damage. Embedded in a finite element environment, this new model provides a useful tool to define safe loading regimes for arterial tissue, which could be used to inform computer-enhanced surgical systems to minimise tissue damage in robotic surgery and, in general, to optimise clamp design towards minimal trauma.

\section{Physiology of the healthy artery}

An artery consists of three distinct layers. In healthy arterial tissue, the inner layer, or intima, consists of an endothelial layer. The middle layer, the media, is the most important load-bearing layer of the artery within the physiological loading domain. It consists of collagen, elastin and smooth muscle cells separated by fenestrated elastic laminae. The outer layer, the adventitia, is surrounded by loose connective tissue. It consists mainly of thick bundles of collagen fibres arranged in a helical structure (Rhodin 1979). For a more detailed description of arterial wall morphology, the reader is referred to, for example, Rhodin (1979) and Holzapfel and Ogden (2010b).

Arterial blood pressure is regulated acutely by altering the luminal diameter, which is controlled by balancing vasoconstricting and vasodilating influences on the smooth muscle cells in a mechanochemical process. Smooth muscle cells contain actin and myosin filaments that slide relative to each other, causing contraction and relaxation. This relative sliding is accomplished by configurational changes of the crossbridges, or myosin heads, that connect the myosin to the actin filament. These configurational changes are caused by phosphorylation and dephosphorylation of the myosin heads, as a function of the intracellular calcium concentration. For a detailed description of the mechanochemical process of smooth muscle cell contraction, the reader is referred to, for example, Stålhand et al. (2008) or Murtada et al. (2010).

\section{Material modelling}

Constitutive models characterise the mechanical behaviour of materials through a functional relation between stresses and strains. A great number of models for cardiovascular tissue exist, aimed at capturing its specific features (Vito and Dixon 2003; Göktepe et al. 2011). For an overview of constitutive models for cardiovascular tissue, or for biological soft tissue in general, the reader is referred to, for example, Gasser et al. (2006); Famaey and Vander Sloten (2008). Holzapfel et al. (2000) have introduced one of the most commonly used hyperelastic, anisotropic material models for arteries, which accounts for two collagen fibre families along two symmetrically arranged directions and allows for a certain amount of dispersion. This model nicely captures the typical nonlinear behaviour as wavy collagen fibres are gradually recruited when the tissue is stretched. In this baseline model, however, the material behaves completely passive, that is, the model does not account for the contractile nature of the smooth muscle cells present in the arterial wall.

The first mechanical representation of a muscle was proposed by Hill (1938), which was extended to the three-element Hill model by Fung (1970). This model consists of a contractile element in series with a spring element, representing the contractile unit. Another spring in parallel represents the surrounding material. For smooth muscle, Gestrelius and Borgström (1986) proposed a variation of the three-element Hill model. Yang et al. (2003) were the first to couple the mechanical representation to an electrochemical model by Hai and Murphy (1988), incorporating the calcium-driven configurational changes of the cross-bridges. This approach was also followed and improved for situations with large deformations, by Stålhand et al. (2008), Murtada et al. (2010), Kroon (2010) and Schmitz and Böl (2011). However, so far, the active contribution of smooth muscle has not yet been combined with the collagen fibre contribution, nor have the models been implemented in a finite element framework. The model proposed by Zulliger et al. (2004) does combine the active contribution with a stochastic collagen fibre contribution in a pseudoelastic-type strain energy function. In Göktepe and Kuhl (2010) and Rausch et al. (2011), finite element formulations were proposed in which mechanical contraction was controlled via electrical and chemical fields, respectively. Unfortunately, these models are phenomenological and thus less straightforward to populate with realistic experiment-based material parameters. In this article, the active contribution by Murtada et al. (2010) will be combined with the collagen fibre contribution by Holzapfel et al. (2000) and implemented in a finite element framework to account for tissue heterogeneity. Moreover, the material parameters related to the active constituent will be calibrated for rat abdominal arteries by means of in vivo experiments.

Most existing material models are designed to describe the material in its physiological state. These models, however, fail to capture damage mechanisms that may occur when the tissue is loaded in the sub- or supra-physiological domain, for example, during surgical manipulation. Motivated by the typical stress softening or Mullins effect in rubber-like materials, Simo and Ju (1987) introduced a discontinuous damage model that allows progressive degradation of an isotropic material to be captured. Balzani et al. (2006) have adapted this approach to describe damage to arterial tissue based on the Holzapfel-material model. Other approaches 
exist to model damage in rubber-like materials, in a continuous manner (Miehe 1995), or pseudoelastically (Ogden and Roxburgh 1999). Dargazany and Itskov (2009) proposed a network evolution model to model anisotropic damage in rubber which was later applied for biological tissues by Ehret and Itskov (2009). Hokanson and Yazdani (1997) incorporated anisotropic damage in arteries by weighting an Ogdentype strain energy function with a fourth order damage tensor. Another suggestion for anisotropic damage to arterial tissue controlled by material constants was made in Volokh (2008, 2011). Also for arterial tissue, damage to the collagen fibres has been described in a stochastic, worm-like chain model by Rodríguez et al. (2006). From the same group, Calvo et al. (2007) presented a continuum damage model with discontinuous softening in matrix and collagen fibres. Viscoelasticity was introduced in these damage models by Pena et al. (2010). These damage models, however, neither include the active smooth muscle contribution nor the damage to the smooth muscle cells. In this article, damage will be incorporated in a manner similar to Balzani et al. (2006), this time including the contributions of healthy and potentially damaged smooth muscle cells.

\section{Experimental characterisation}

Every constitutive model introduces a set of material parameters that needs to be calibrated for the particular type of tissue. Specific experimental setups, such as uniaxial and biaxial tensile tests or extension-inflation tests can be performed to calibrate the material parameters for standard passive hyperelastic models, as described, for example, in Sacks and Sun (2003), Holzapfel and Ogden (2010a).

To quantify the active response of the smooth muscle, isometric and/or isotonic contraction experiments can be performed ex vivo, as described in Barone et al. (1989), Gleason et al. (2004), Murtada et al. (2010) and Böl et al. (2012). Recently, Itoh et al. (2009) and Tsamis et al. (2011) have reported in vivo experiments to identify active muscle force in cardiovascular tissue in situ.

Damage is frequently assessed through the evaluation of histological images of the tissue, for example in Hsi et al. (2002), Manchio et al. (2005) and De et al. (2007). For example, live-dead stains can help to identify cell viability, and $\mathrm{H}$ and $\mathrm{E}$ (haematoxylin and eosin) and collagen stainings can visualise ruptures in the collagen fibres. Unfortunately, most studies of tissue damage are qualitative in nature, both in the application of the tissue load to induce the damage and in the subsequent damage assessment. To calibrate the damage material parameters, however, quantitative experiments are essential. De et al. (2007) were the first to characterise damage quantitatively for porcine liver. For cardiovascular tissue, previous work (Famaey et al. 2010) reports on a study in which the damage to the smooth muscle cells of rat abdominal arteries is quantitatively assessed in an isometric contraction test after in vivo clamping to well-defined loading levels. In this article, this quantitative damage information will be used to calibrate the parameters of the new material model.

\section{Outline}

Section 2 introduces our new material model, accounting for the three major tissue constituents: extracellular matrix, collagen and smooth muscle cells. In particular, we allow each constituent to degrade independently. The features of the model are first illustrated in a simple homogeneous uniaxial cyclic extension and compression test in Sect. 3. Section 4 then demonstrates how the model can be applied to predict smooth muscle cell damage in rat abdominal arteries through clamping and how the damage parameters can be identified using actual experiments. Section 5 discusses the presented model and suggests further directions for future work.

\section{Governing equations for arteries}

Through an additive decomposition of the strain energy, the following constitutive model for active healthy and degraded arterial tissue characterises the properties of (i) an isotropic matrix material constituent, (ii) an anisotropic constituent attributed to the dispersed collagen fibres and (iii) an anisotropic smooth muscle cell constituent. The first two constituents are motivated by the Holzapfel-material model as proposed in Holzapfel et al. (2000), whereas the third component is motivated by the mechanical smooth muscle-activation model described by Murtada et al. (2010). The damage accumulating in the different constituents during mechanical loading is characterised through a strain energy-driven damage function for each individual constituent, motivated by the formulation by Balzani et al. (2006). In the remainder of the article, the model will be referred to as the three-constituent damage model.

\subsection{Kinematic prerequisites}

Since soft biological tissues can undergo large physiological deformations, the key kinematic quantity to characterise the deformation process is the deformation gradient $\mathbf{F}$, that is, the gradient of the deformation map $\varphi$ with respect to the undeformed position $\boldsymbol{X}$ :

$\mathbf{F}=\nabla_{X} \boldsymbol{\varphi} \quad$ and $\quad J=\operatorname{det}(\mathbf{F})$.

Here, $J$ denotes its Jacobian $J$, which is close to one, $J \approx 1$, for nearly incompressible materials. In that case, it proves convenient to decompose the deformation gradient into a deviatoric part, $\overline{\mathbf{F}}$, and a volumetric part, $J^{1 / 3} \mathbf{I}$,
248 
$\mathbf{F}=J^{1 / 3} \overline{\mathbf{F}}$

Typically, the deformation of incompressible materials is characterised in terms of the invariants of the deviatoric part $\overline{\mathbf{C}}$ of the right Cauchy-Green tensor $\mathbf{C}$, with

$\mathbf{C}=\mathbf{F}^{\mathrm{T}} \mathbf{F} \quad$ and $\quad \overline{\mathbf{C}}=\overline{\mathbf{F}}^{\mathrm{T}} \overline{\mathbf{F}}$.

The basic deviatoric invariants $\bar{I}_{i}$ take the following explicit representation:

$\bar{I}_{1}=\operatorname{tr}(\overline{\mathbf{C}})$,

$\bar{I}_{2}=\frac{1}{2}\left[\operatorname{tr}^{2}(\overline{\mathbf{C}})-\operatorname{tr}\left(\overline{\mathbf{C}}^{2}\right)\right]$,

$\bar{I}_{3}=\operatorname{det}(\overline{\mathbf{C}})$.

While the basic invariants characterise the isotropic material behaviour, the anisotropic invariants $\bar{I}_{4}^{\text {fib }}, \bar{I}_{6}^{\text {fib }}$, and $\bar{I}_{4}^{\text {smc }}$ characterise the stretches along the fibre and smooth muscle cell directions, see Gasser et al. (2006):

$\bar{I}_{4}^{\mathrm{fib}}=\lambda_{\theta}^{2} \cos ^{2} \alpha^{\mathrm{fib}_{1}}+\lambda_{z}^{2} \sin ^{2} \alpha^{\mathrm{fib}}{ }_{1}$,

$\bar{I}_{6}^{\mathrm{fib}}=\lambda_{\theta}^{2} \cos ^{2} \alpha^{\mathrm{fib}_{2}}+\lambda_{z}^{2} \sin ^{2} \alpha^{\mathrm{fib}_{2}}$,

$\bar{I}_{4}^{\text {smc }}=\lambda_{\theta}^{2} \cos ^{2} \alpha^{\text {smc }}+\lambda_{z}^{2} \sin ^{2} \alpha^{\text {smc }}$

Here, $\lambda_{\theta}$ and $\lambda_{z}$ are the stretches in the circumferential and axial directions, respectively. Moreover, $\alpha^{\mathrm{fib}_{1}}, \alpha^{\mathrm{fib}_{2}}$ and $\alpha^{\text {smc }}$ denote the angles between the circumference and the mean directions of the fibre and smooth muscle families. In the case of arteries, two fibre families are oriented symmetrically with respect to the cylinder axis, so that $\alpha^{\mathrm{fib}_{1}}=-\alpha^{\mathrm{fib}_{2}}$ and, consequently, $\bar{I}_{4}^{\text {fib }}=\bar{I}_{6}^{\text {fib }}$. Finally, the pseudo-invariants $I_{4}^{\mathrm{fib} \star}$ and $I_{6}^{\mathrm{fib} \star}$ are introduced to account for dispersion,

$I_{4}^{\mathrm{fib} \star}=\kappa \bar{I}_{1}+[1-3 \kappa] \bar{I}_{4}^{\mathrm{fib}}$,

$I_{6}^{\text {fib }}=\kappa \bar{I}_{1}+[1-3 \kappa] \bar{I}_{6}^{\text {fib }}$,

where the fibre dispersion $\kappa$ characterises the degree of anisotropy varying from $\kappa=0$ in the anisotropic non-dispersed state to $\kappa=\frac{1}{3}$ in the isotropic state.

\subsection{Constitutive equations}

Since the tissue is assumed to be nearly incompressible, it is common to additively decompose the strain energy function $\Psi$

$\Psi=\Psi^{\mathrm{vol}}+\Psi^{\mathrm{dev}}=\Psi^{\mathrm{vol}}+\Psi^{\mathrm{mat}}+\Psi^{\mathrm{fib}_{1}}+\Psi^{\mathrm{fib}_{2}}+\Psi^{\mathrm{smc}}$,

into a volumetric $\Psi^{\mathrm{vol}}$ and a deviatoric $\Psi^{\mathrm{dev}}$ part. The latter consists of an isotropic contribution of the matrix material $\Psi^{\text {mat }}$, an anisotropic contribution of two families of collagen fibres $\Psi^{\text {fib }_{1}}$ and $\Psi^{\text {fib }}$, and a contribution of the smooth muscle cells $\Psi^{\text {smc }}$. The individual contributions will be specified in detail in the following section. All deviatoric components are allowed to undergo degradation in the case of physiological overload. Simo and Ju (1987) in general and Balzani et al. (2006) for arteries have described the approach of weighting the strain energy with a scalar valued damage variable $[1-d]$. This model builds upon the classical damage concept, and introduces an independent damage variable for each individual constituent.

\section{Volumetric bulk material}

299 300 301

The volumetric free energy $\Psi^{\mathrm{vol}}$ can, for example, be expressed as follows (Arruda and Boyce 1993):

$\Psi^{\mathrm{vol}}=\Lambda\left[\frac{1}{2}\left[J^{2}-1\right]-\ln (J)\right]$.

The penalty parameter $\Lambda$ corresponds to $\kappa / 2$, with $\kappa$ the bulk modulus (in $\mathrm{MPa}$ ), and should be set high enough to ensure near-incompressibility.

Since this term is handled separately in an incompressible finite element formulation, we will now focus on the four contributions to the deviatoric energy $\Psi^{\mathrm{dev}}$, which are the primary descriptors of the material behaviour.

\section{Extracellular matrix}

The extracellular matrix is characterised through an isotropic free energy $\Psi^{\text {mat }}$, which is allowed to degrade according to the classical damage concept:

$\Psi^{\mathrm{mat}}=\left[1-d^{\mathrm{mat}}\right] \widehat{\Psi}^{\mathrm{mat}}$.

Here, $\widehat{\Psi}^{\text {mat }}$ denotes the elastic energy of the extracellular matrix:

$\widehat{\Psi}^{\mathrm{mat}}=\frac{1}{2} c\left[\bar{I}_{1}-3\right]$,

where $c>0$ characterises the matrix stiffness (in $\mathrm{kPa}$ ). The evolution of the damage variable of the extracellular matrix $d^{\text {mat }}$ is driven by the undamaged elastic extracellular matrix energy, as proposed by Balzani et al. (2006):

$d^{\mathrm{mat}}=\gamma^{\mathrm{mat}}\left[1-\exp \left(-\beta^{\mathrm{mat}} / m^{\mathrm{mat}}\right)\right]$.

The weighting factor $\gamma^{\text {mat }}$ (in $\mathrm{kPa}$ ) can be used to tune the sensitivity to damage, $\left.\left.\gamma^{\text {mat }} \in\right] 0,1\right]$, or to turn the damage off altogether, $\gamma^{\text {mat }}=0 . m^{\text {mat }}$ is a dimensionless parameter of the damage model. The variable $\beta^{\text {mat }}$ is an internal variable keeping track of the maximum elastic strain energy experienced so far, within the time interval $0 \leq t \leq \tau$ (Balzani et al. 2006):

$\beta^{\mathrm{mat}}=\max _{0 \leq t \leq \tau}\left(\widehat{\Psi}^{\mathrm{mat}}(t)-\Psi_{0}^{\mathrm{mat}}\right)$.

Since it can be assumed that no damage occurs in the physiological range, the damage threshold $\Psi_{0}^{\text {mat }}$ is initialised with the strain energy in the extracellular matrix at systolic pressure. For heterogeneous problems, $\Psi_{0}^{\text {mat }}$ may therefore differ for each material point, and is thus not strictly a material property. 


\section{Collagen fibres}

Collagen fibres will only contribute when under tension. Similar to the free energy of the matrix, the free energy of the collagen fibres accounts for both an elastic and a degrading response,

$\Psi^{\mathrm{fib}_{i}}=\left[1-d^{\mathrm{fib} i}\right] \widehat{\Psi}^{\mathrm{fib}_{i}} \quad i=1,2$,

where the energy contributions of the two families of collagen fibres are formulated according to Gasser et al. (2006):

$\widehat{\Psi}^{\mathrm{fib}_{i}}=\frac{k_{1}}{2 k_{2}}\left[\exp \left(k_{2}\left[I_{i}^{\mathrm{fib} \star}-1\right]^{2}\right)-1\right]$.

Here, $k_{1}>0$ characterises the fibre stiffness (in $\mathrm{kPa}$ ) and $k_{2}>0$ is a dimensionless parameter. Damage of the two fibre families $d^{\text {fib }_{i}}$ can again be described in terms of the elastic fibre energies $\widehat{\Psi}^{\mathrm{fib}_{i}}$ (Balzani et al. 2006):

$d^{\mathrm{fib}_{i}}=\gamma^{\mathrm{fib}}\left[1-\exp \left(-\beta^{\mathrm{fib}_{i}} / m^{\mathrm{fib}}\right)\right]$,

where $\gamma^{\text {fib }}$ and $m^{\text {fib }}$ are the two fibre damage parameters and $\beta^{\text {fib }_{i}}$ are the internal variables of each fibre family keeping track of the maximum value of the elastic fibre energies experienced so far (Balzani et al. 2006):

$\beta^{\mathrm{fib}_{i}}=\max _{0 \leq t \leq \tau}\left(\widehat{\Psi}^{\mathrm{fib}_{i}}(t)-\Psi_{0}^{\mathrm{fib}}\right)$.

Again, the damage threshold $\Psi_{0}^{\text {fib }}$ is initialised with the strain energy of the fibres at systolic pressure and may therefore differ for each material point. Since the internal variables $\beta^{\text {fib }_{i}}$ are driven by the elastic strain energies $\widehat{\Psi}^{\mathrm{fib}_{i}}$, material degradation will only take place when the fibres are under tension, as the strain energy is zero when in compression.

\section{Smooth muscle cells}

The smooth muscle cells form an integral part of the matrix constituent, even in their passive state. Therefore, their degradation is assumed to depend on both the passive damage

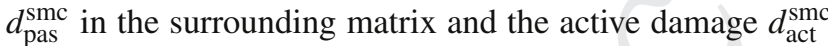
in the smooth muscle cells themselves:

$\Psi^{\mathrm{smc}}=\left[1-d_{\mathrm{pas}}^{\mathrm{smc}}\right]\left[1-d_{\mathrm{act}}^{\mathrm{smc}}\right] \widehat{\Psi}^{\mathrm{smc}}$.

In the undamaged state, the energy of the smooth muscle cells $\widehat{\Psi}^{\text {smc }}$ can be expressed as follows (Murtada et al. 2010):

$\widehat{\Psi}^{\mathrm{smc}}=\frac{1}{2} \mu^{\mathrm{smc}}\left[n_{\mathrm{III}}+n_{\mathrm{IV}}\right]\left[\sqrt{I_{4}^{\mathrm{smc}}}+u_{\mathrm{rs}}-1\right]^{2}$,

where $\mu^{\mathrm{smc}}$ characterises the stiffness of the actin-myosin filament apparatus (in $\mathrm{kPa}$ ). The kinetics of the actin-myosin power stroke are modelled through a four-state model described by Hai and Murphy (1988) and adopted by Murtada et al. (2010), Kroon (2010) and Stålhand et al. (2011). This model describes the transitions between the four states
$n_{\mathrm{I}}, n_{\mathrm{II}}, n_{\mathrm{III}}$ and $n_{\mathrm{IV}}$ of the myosin heads as a function of the calcium concentration as follows:

$$
\left[\begin{array}{c}
\dot{n}_{\mathrm{I}} \\
\dot{n}_{\mathrm{II}} \\
\dot{n}_{\mathrm{III}} \\
\dot{n}_{\mathrm{IV}}
\end{array}\right]=\left[\begin{array}{cccc}
-\kappa_{1} & \kappa_{2} & 0 & \kappa_{7} \\
\kappa_{1}-\left(\kappa_{2}+\kappa_{3}\right) & \kappa_{4} & 0 \\
0 & \kappa_{3} & -\left(\kappa_{4}+\kappa_{5}\right) & \kappa_{6} \\
0 & 0 & \kappa_{5} & -\left(\kappa_{6}+\kappa_{7}\right)
\end{array}\right]\left[\begin{array}{c}
n_{\mathrm{I}} \\
n_{\mathrm{II}} \\
n_{\mathrm{III}} \\
n_{\mathrm{IV}}
\end{array}\right]
$$

Here, $n$ are the fractions of the four states, which sum up 386 to one, $\sum n_{i}=1$. The $\kappa_{i}\left(\right.$ in $\left.\mathrm{s}^{-1}\right)$ are the rate constants of ${ }_{387}$ the model, where $\kappa_{1}$ and $\kappa_{7}$ are a function of the calcium 388 concentration. In particular, $n_{\mathrm{I}}$ and $n_{\mathrm{II}}$, are the fractions of 389 dephosphorylated and phosphorylated myosin heads that are not attached to the actin filament, and thus not mechanically contributing. $n_{\mathrm{III}}$ and $n_{\mathrm{IV}}$ are the fractions of phosphorylated and dephosphorylated myosin heads, or cross-bridges, attached to the actin filaments, and thus contributing to the stiffness. The power stroke occurs through a conformational change in state III, after which the myosin heads transform back into state II. As long as the myosin heads remain phosphorylated, they cycle back and forth between states II and III, thus generating contraction. In state IV, the myosin heads are still attached to the actin filament but dephosphorylated and thus unable to perform a power stroke.

In Eq. (18), $u_{\mathrm{rs}}$ is the average normalised relative sliding between the myosin and the actin filaments. It follows a viscous evolution law:

$\dot{u}_{\mathrm{rs}}=\frac{1}{\eta}\left[P^{\mathrm{smc}}-P^{\mathrm{mat}}\right]$,

where $\eta$ is a viscosity parameter (in MPa s), $P^{\text {smc }}$ denotes the active stress exerted by the attached myosin heads and $P^{\text {mat }}$ denotes the stress from the surrounding matrix. The active stress $P^{\text {smc }}$ can be approximated by the following step function:

$P^{\mathrm{smc}}= \begin{cases}\kappa_{c} n_{\mathrm{III}} & \text { for } P^{\mathrm{mat}}<\kappa_{c} n_{\mathrm{III}} \\ P^{\mathrm{mat}} & \text { else } \\ \kappa_{c}\left[n_{\mathrm{III}}+n_{\mathrm{IV}}\right] & \text { for } \kappa_{c}\left[n_{\mathrm{III}}+n_{\mathrm{IV}}\right]<P^{\mathrm{mat}}\end{cases}$

where $\kappa_{C}$ is a material parameter (in $\mathrm{MPa}$ ) related to the driving force per myosin head, see Murtada et al. (2010) and Kroon (2010) for details. Smooth muscle cell degradation is governed by two damage variables, $d_{\mathrm{pas}}^{\mathrm{smc}}$ characterising the damage to the surrounding matrix and $d_{\mathrm{act}}^{\mathrm{smc}}$ characterising the damage to the smooth muscle cells themselves:

$d_{\text {pas }}^{\text {smc }}=\gamma_{\text {pas }}^{\text {smc }}\left[1-\exp \left(-\beta^{\text {mat }} / m_{\text {pas }}^{\text {smc }}\right)\right]$,

$d_{\mathrm{act}}^{\mathrm{smc}}=\gamma_{\mathrm{act}}^{\mathrm{smc}}\left[1-\exp \left(-\beta^{\mathrm{smc}} / m_{\mathrm{act}}^{\mathrm{smc}}\right)\right]$.

The internal variable for matrix damage $\beta^{\text {mat }}$ is defined in Eq. (12), and the internal variable for smooth muscle cell damage $\beta^{\text {smc }}$ is defined as:

$\beta^{\mathrm{smc}}=\max _{0 \leq t \leq \tau}\left(\widehat{\Psi}^{\mathrm{smc}}(t)-\Psi_{0}^{\mathrm{smc}}\right)$. 390 391 392 393 394 395 396 397 398 399 400 401 402 403 
Both keep track of the loading history through the maximum value of the elastic matrix and smooth muscle cell energies experienced so far.

In the present application, damage values are relatively low and no localised deformation has been observed. To avoid the loss of uniqueness of the underlying boundary value problem in the context of larger damage values, we recommend the use of gradient enhanced damaged models, see Kuhl and Ramm (1999), Mahnken and Kuhl (1999) for details.

In general, it would be possible to also include viscous effects. However, viscosity plays a rather minor role in arterial clamping. Firstly, in view of the application of tissue overload prevention in surgery, an overestimation is more acceptable than an underestimation. Not including viscosity will result in an overestimation of the loading. Secondly, during surgery, the typical movements of a surgeon are at a rather low frequency of maximally $2 \mathrm{~Hz}$.

\section{Computational modelling of arteries}

This section addresses the implementation of the arterial model into the finite element programme Abaqus.

\subsection{Implementation}

The constitutive model is implemented in the Abaqus user subroutine UANISOHYPER_INV, a family of subroutines designed for anisotropic, hyperelastic material models, in which the strain energy density function $\Psi$ is formulated as a function of the strain invariants. This subroutine can handle and update solution-dependent internal variables and requires that the derivatives of the strain energy function are defined with respect to the scalar invariants $\bar{I}_{1}, \bar{I}_{2}, \bar{I}_{3}, \bar{I}_{4}^{\text {fib }}, \bar{I}_{6}^{\text {fib }}, \bar{I}_{4}^{\text {smc }}$, which are provided as input. It is called at each integration point during each load increment to calculate the total strain energy $\Psi$ and its first and second derivatives with respect to the invariants $\partial \Psi / \partial \bar{I}_{i}$ and $\partial^{2} \Psi / \partial \bar{I}_{i} \partial \bar{I}_{j}$ for $i, j=1,2,3,4^{\text {fib }}, 6^{\text {fib }}, 4^{\text {smc }}$.

Through the input file, a local coordinate system must be set, containing the local directions $\alpha^{\text {fib }}$ for the collagen fibres and $\alpha^{\text {smc }}$ for the smooth muscle cells. When defining the material, memory must be allocated for nine solution-dependent state variables, namely the damage driving forces $\beta^{\text {mat }}, \beta^{\mathrm{fib}_{1}}, \beta^{\mathrm{fib}_{2}}$, and $\beta^{\mathrm{smc}}$, and the damage thresholds $\Psi_{0}^{\mathrm{mat}}, \Psi_{0}^{\mathrm{fib}}, \Psi_{0}^{\mathrm{fib}}$, and $\Psi_{0}^{\mathrm{smc}}$. The ninth state-dependent variable is the relative sliding $u_{\mathrm{rs}}$ in the actin-myosin complex, which needs to be stored because of its viscous nature.

The anisotropic, hyperelastic, user-defined material model must be specified with all the material parameters described above, choosing the options ' formulation = invariant', 'local directions $=3$ ' and 'type $=$
Table 1 Parameter sets for cyclic uniaxial tension and compression test in Sect. 3.2

\begin{tabular}{lllll}
\hline Parameter & Var 1 & Var 2 & Var 3 & Var 4 \\
\hline$\mu^{\text {smc }}$ & $0.0 \mathrm{kPa}$ & $0.0 \mathrm{kPa}$ & $0.2 \mathrm{kPa}$ & $0.2 \mathrm{kPa}$ \\
$\gamma^{i}$ & $0.0(-)$ & $0.9(-)$ & $0.0(-)$ & $0.9(-)$ \\
\hline
\end{tabular}

$i=$ mat, fib, $\mathrm{smc}_{\mathrm{pas}}, \mathrm{smc}_{\mathrm{act}}$

All other material parameters can be found in Table 2

incompressible'. A conceptual drawback of the UANISOHYPER_INV subroutine is that it does not provide access to the time step of the solution process, which should be known for correct programming of the viscous evolution law described in Eq. (20). This implies that the exact time step is only known if a fixed time increment is set, by adding the option 'direct' to the keyword 'static' in the input file. Otherwise, only the minimum and maximum allowable time step can be externally prescribed.

3.2 Model problem of cyclic uniaxial tension and compression

The new constitutive model was tested for the simple model problem of cyclic uniaxial tension and compression using a hexahedral C3D8H element. Homogeneous boundary conditions were applied, namely a gradually increasing, sawtooth stretch pattern, as shown in Fig. 1. To explore the parameter sensitivity of the model, four different sets of material parameters were compared in tension by altering the smooth muscle cell stiffness $\mu^{\text {smc }}$ and the damage weighting factor $\gamma^{i}$, see Table 1 . All other parameters were selected according to the rationale explained in Sect. 4.2 as shown in Table 2.

As a first benchmark test, the three-constituent damage model was compared with the Abaqus implementation of the standard Holzapfel-Gasser-Ogden model, where the smooth muscle cell stiffness $\mu^{\mathrm{smc}}$ and the damage weighting factors $\gamma^{i}$ were set to zero (variation 1). Both simulations yielded exactly the same results, verifying the correct implementation of the baseline model. Next, different features of the model were gradually added and evaluated for consistency. Figure 2 shows the stress-strain curves for the prescribed loading pattern from Fig. 1 for four variations of the new material model in tension and two variations in compression. By turning off the smooth muscle contribution $\mu^{\text {smc }}$ and the damage $\gamma^{i}=0$ in variation 1 , the model captures the Holzapfel-Gasser-Ogden material by Abaqus as a special case. When the damage material parameter $\gamma^{i}$ is increased to 0.9 $(-)$ in variation 2 , the dashed red curve is obtained, showing the progressive failure of the fibres and matrix material. When the smooth muscle stiffness $\mu^{\mathrm{smc}}$ is increased to a value of $0.2 \mathrm{MPa}$ in variation 3 , the solid green curve is obtained. It shows how, in the fully contracted state, the smooth muscle 
Table 2 Parameters used in the finite element model

\begin{tabular}{|c|c|c|}
\hline Parameter & Value & Source \\
\hline \multicolumn{3}{|c|}{ Matrix material } \\
\hline$c$ & $23.63 \mathrm{kPa}$ & Famaey et al. (2012) \\
\hline$\gamma^{\text {mat }}$ & $0(-)$ & Not studied \\
\hline$m^{\text {mat }}$ & $/ \mathrm{kPa}$ & Not studied \\
\hline \multicolumn{3}{|c|}{ Collagen fibres } \\
\hline$\alpha^{\mathrm{fib}}$ & $\pm 5^{\circ}$ & O'Connell et al. (2008) \\
\hline$k_{1}$ & $32.51 \mathrm{kPa}$ & Famaey et al. (2012) \\
\hline$k_{2}$ & $3.05(-)$ & \\
\hline$\kappa$ & $0.16(-)$ & \\
\hline$\gamma^{\text {fib }}$ & $0(-)$ & Not studied \\
\hline$m^{\mathrm{fib}}$ & $/ \mathrm{kPa}$ & Not studied \\
\hline \multicolumn{3}{|c|}{ Smooth muscle cells_chemical rate constants } \\
\hline$\kappa_{1}, \kappa_{6}$ & $0.14 \mathrm{~s}^{-1}$ & Hai and Murphy (1988) \\
\hline$\kappa_{2}, \kappa_{5}$ & $0.5 \mathrm{~s}^{-1}$ & \\
\hline$\kappa_{3}, 4 \kappa_{4}$ & $0.44 \mathrm{~s}^{-1}$ & \\
\hline$\kappa_{7}$ & $0.01 \mathrm{~s}^{-1}$ & \\
\hline \multicolumn{3}{|c|}{ Smooth muscle cells-mechanical constants } \\
\hline$\mu^{\mathrm{smc}}$ & $0.25 \mathrm{MPa}$ & Fitted to experiments \\
\hline$\kappa_{c}$ & $0.93 \mathrm{MPa}$ & Fitted to experiments \\
\hline$\eta$ & $60 \mathrm{MPa} \mathrm{s}$ & Murtada et al. (2010) \\
\hline$\alpha^{\mathrm{smc}}$ & $0^{\circ}$ & O’Connell et al. (2008) \\
\hline$\gamma_{\text {act }}^{\text {smc }}$ & $0(-)$ & Not studied \\
\hline$m_{\text {act }}^{\text {smc }}$ & $/ \mathrm{kPa}$ & Not studied \\
\hline$\gamma_{\text {pas }}^{\text {smc }}$ & $0.9(-)$ & Fitted to experiments \\
\hline$m_{\text {pas }}^{\text {smc }}$ & $0.03 \mathrm{kPa}$ & Fitted to experiments \\
\hline
\end{tabular}

cells actively contribute to the stiffness. A slight effect of the contractile element can be observed. When the damage material parameter $\gamma^{i}$ is increased to $0.9(-)$ in variation 4 , the solid red curve with arrows is obtained, clearly demonstrating the progressive smooth muscle cell degradation as well as the degradation of the fibres and the smooth muscle cells. By increasing or decreasing the damage weighting factor $\gamma^{i}$ within the range $0<\gamma^{i}<1$, the solid red curve with arrows decreases or increases, respectively, bounded from above and below by the solid green and dashed blue lines.

The solid black curve is obtained when loading variation 1 or variation 3 of the model in compression. In this regime, the smooth muscle cells do not contribute and the fibres contribute only very slightly due to their small angle with respect to the loading direction. The grey curve, finally, is obtained when variations 2 or 4 are loaded in compression. Again, progressive degradation (of the matrix material) can be observed. Note that in these last two curves the absolute values of the stress and the strain are provided.

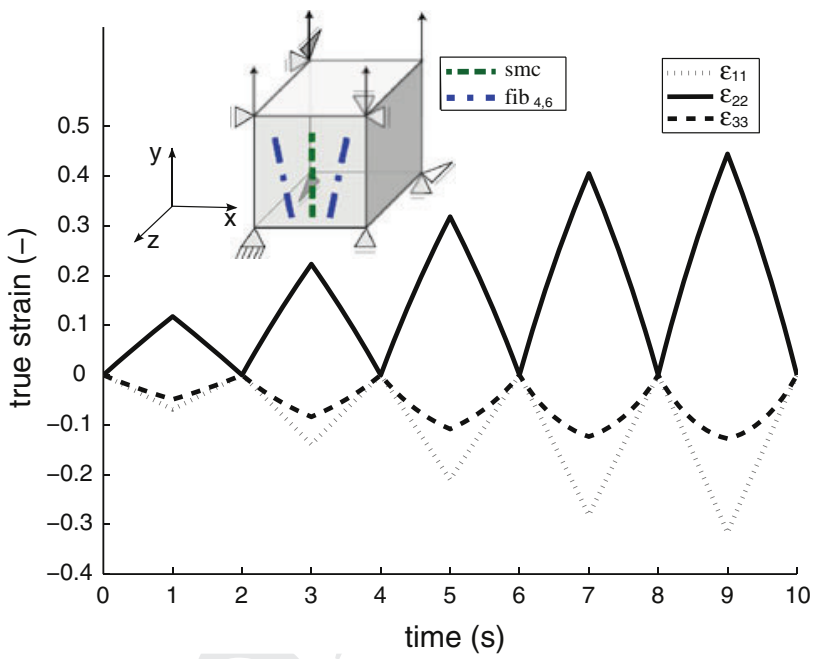

Fig. 1 Strain profile for homogeneous cyclic uniaxial tension and compression test. $\epsilon_{11}, \epsilon_{22}$ and $\epsilon_{33}$ are the strains in the three principal directions. The lines in the block depict the average direction of the two collagen fibre families and the smooth muscle cells

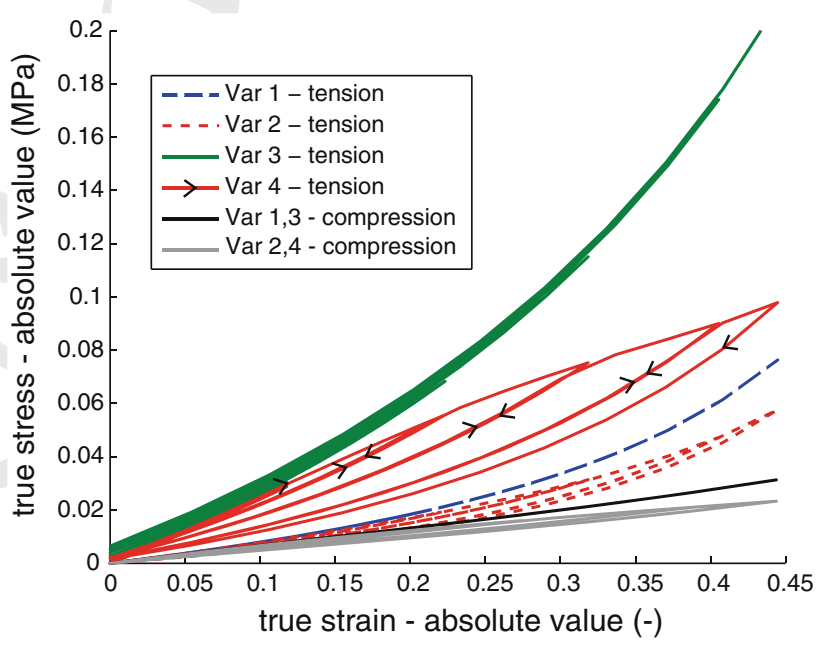

Fig. 2 Stress-strain curve for a homogeneous cyclic uniaxial tension test and a compression test. Curves correspond to healthy smooth muscle, fibres and matrix material (solid green), progressively damaging smooth muscle, fibres and matrix (solid red with arrows), no smooth muscle with healthy fibres and matrix (dashed blue), and progressively damaging fibres and matrix (dashed red), all in tension. The solid black curve corresponds to healthy material in compression, and the solid grey curve to progressively damaging material in compression. Note that the absolute values of the stress and the strain are provided. The prescribed loading profile is shown in Fig. 1. The different sets of material parameters are summarised in Table 1

\section{Smooth muscle cell damage through clamping}

The three-constituent damage model is put to use to simulate the damage process occurring during the clamping of a rat abdominal artery. To test the realism of the model, the results were compared with actual experiments, more thoroughly described in Famaey et al. (2010), in which the abdominal 


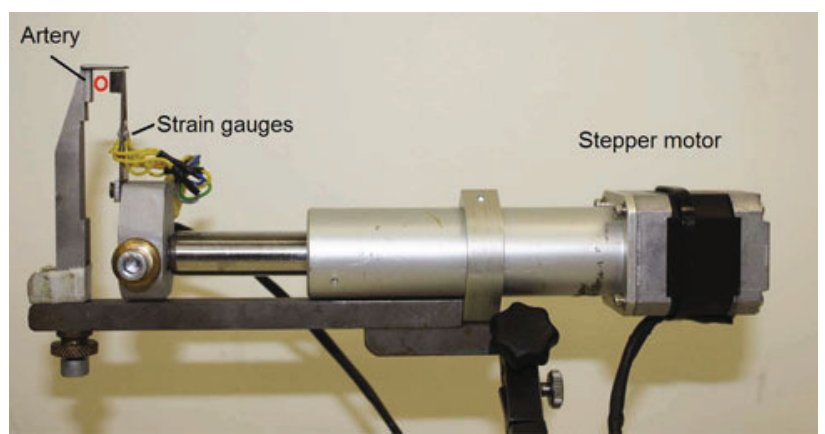

Fig. 3 Mechanical clamping device

arteries of rats were clamped up to a defined clamping force. Subsequently, to quantify the degradation of the smooth muscle cells, the contracting capability of the clamped segment was measured in a myograph as explained in Sect. 4.1. Both experimental processes, that is, arterial clamping and subsequent myograph testing, were simulated numerically using the three-constituent damage model as described in Sect. 4.2.

\subsection{Experimental model}

\section{Arterial clamping}

In order to correlate the degree of damage to the degree of mechanical loading to which the tissue was previously subjected, loading should be applied in a controllable way. Ideally, loading should be applied in vivo, so that the induced damage can be solely attributed to the loading and not to nonphysiological ex vivo conditions. Since subsequent damage quantification requires excision of tissue, undamaged control segments should also be excised and tested as controls, to rule out damage due to the excision process. To clamp the artery in a controlled way, a hand-held mechanical device, shown in Fig. 3, was designed that allows clamping of a rat abdominal artery in an in vivo setting to a known force, measured with strain gauges on the clamping arms (Famaey et al. 2010).

\section{Functional damage assessment}

One damage quantification method is to compare the degree of functionality of a damaged tissue to that of an intact one. For the specific case of arterial tissue, functionality refers to the vasoregulating capability of the tissue, that is, the potential of the smooth muscle cells to contract or relax in order to regulate the blood pressure. This vasoregulating capability can be quantified in an experimental setup, known as a 'myograph'.

Schematically shown in Fig. 4, the myograph consists of a water-jacketed organ chamber in which an excised cylindrical section of an artery can be mounted. Two rods slide

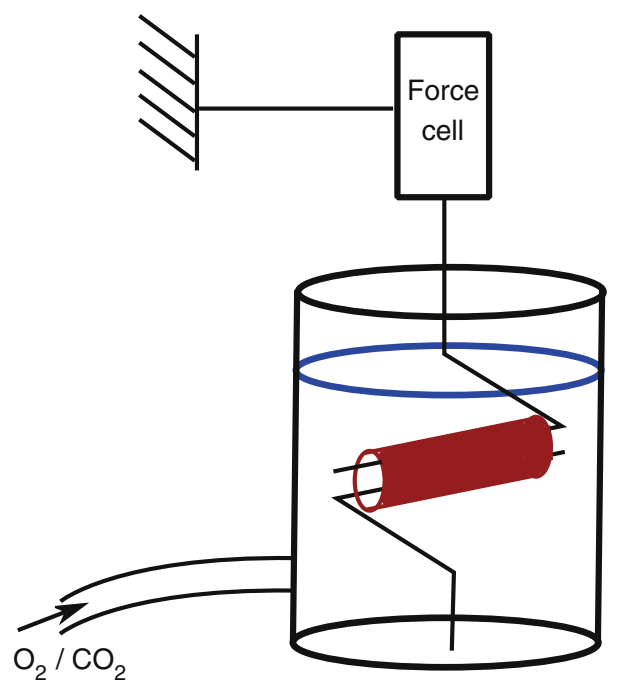

Fig. 4 Custom made functional testing device. Two rods slide into the lumen of the sample, one rod is connected to the base of the set-up, the other to a load cell suspended above the set-up, so that isometric tension can be recorded. The sample is immersed in water-jacketed organ chamber filled with Krebs buffer

into the lumen of the sample, whereby one rod is connected to the base of the setup, and the other to a load cell suspended above the set-up, so that isometric tension can be recorded. The height of the load cell can be manually adjusted to set an optimal preload on the sample. The sample is immersed in a Krebs buffer at $37^{\circ} \mathrm{C}$ and continuously gassed with a mixture of $95 \%$ oxygen and $5 \%$ carbon dioxide. After stabilisation at the optimal preload level, Phenylephrine (PE) at $10^{-6} \mathrm{M}$ is added to the solution to induce contraction. PE is a contracting agent that acts directly on the smooth muscle cells. Sodium nitroprusside (SNP) $\left(10^{-6} \mathrm{M}\right)$ induces an endothelium-independent relaxation so consequently an adequate level of SNP-induced relaxation will indicate intactness of the smooth muscle cells (Callera et al. 2000). Absolute values of relaxation as well as the percentage of relaxation relative to the amount of contraction are recorded and provide a quantitative measure of the damage to the smooth muscle cells when comparing these values to those of an intact sample. More details on the experimental setup can be found in Famaey et al. (2010). A similar custom-designed device to test active force generation in response to electrical stimulation is reported in Böl et al. (2012).

\subsection{Computational model}

\section{Arterial clamping}

A three dimensional finite element model was built in Abaqus/Standard 6.10-2. Here, an idealised cylindrical geometry was used with an outer radius of $0.58 \mathrm{~mm}$, a wall thickness of $0.14 \mathrm{~mm}$ and an initial length of $0.1 \mathrm{~mm}$. These values 


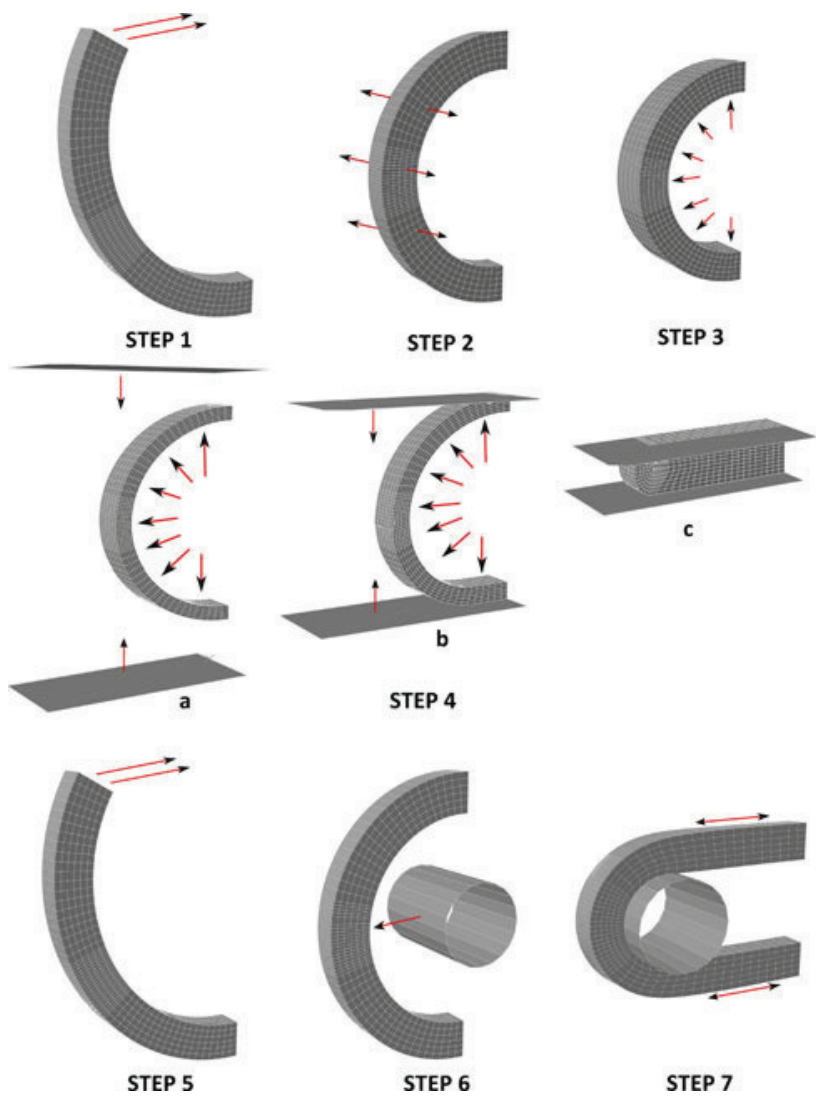

Fig. 5 Schematic overview of the seven steps in the FE simulation representing the loading history of arterial clamping (steps 1-4) and the functional damage assessment (steps 5-7)

were obtained from measurements on rat abdominal arteries described in Famaey et al. (2012). The mesh density was chosen according to the rule of thumb that in bending situations, there should be at least four elements through the thickness. Here, because of severe bending, six instead of four elements were taken across the thickness, and seeding in other dimensions was chosen to ensure regular elements. For the generation of real patient-specific models, we refer to Kuhl et al. (2007) or Balzani et al. (2011). C3D8H elements were assigned to the mesh. The numerical implementation of arterial clamping is subdivided into two steps, (i) the setting of the initial damage level and (ii) the clamping process. Figure 5 shows all steps of the clamping simulation.

In the first part, an opened cylindrical segment with an opening angle of $60^{\circ}$ is closed to account for the circumferential residual stresses (Balzani et al. 2007). Next, the segment is longitudinally stretched by $50 \%$, to account for residual stresses in the longitudinal direction. These values for the residual stresses were obtained from experiments described in Famaey et al. (2012). In the third step, the segment is inflated to an internal pressure of $16 \mathrm{kPa}$. The material model used in this step is the undamaged three-constituent damage model, however, without accumulation of damage. At the end of the third step, the undamaged elastic strain energy of each of the four constituents is written into a matrix of internal or 'solution-dependent variables' for each integration point, using Python scripting. These are the initial damage threshold levels $\Psi_{0}^{\mathrm{i}}$, described in Eqs. (12), (16) and (23) to be used in step 4.

Step 4 starts with a new input file, in which the state of the artery after the first three steps is imported. By importing, the deformations are included as 'initial values' for the model. The solution-dependent variables defined earlier contain the damage threshold levels $\Psi_{0}^{\mathrm{i}}$ specified as 'initial conditions' in the input file. The material model is now updated to enable damage accumulation, $\gamma^{i}>0$, and four extra solution-dependent variables, representing the $\beta^{i}$ described in Eqs. (12), (16) and (23) are added. In addition, two extra parts are added to the assembly of the system, namely an upper and lower clamp, which are gradually moved towards each other during step 4 , until a clamping force of $5 \mathrm{mN}$ is reached. A friction coefficient of $\mu^{\text {clamp }}=0.5$ is used between the clamp and the outer arterial surface. Finally, also the internal pressure boundary conditions are modified to a pulsating pressure between 10 and $16 \mathrm{kPa}$, that gradually decays to zero when the vessel is completely closed. To keep track of the maximum energy level reached for each constituent at every integration point of the system, the four extra solution-dependent variables are updated and stored at each step as internal variables $\beta^{i}$. At the end of the simulation, these solution-dependent variables are again written to a matrix using Python scripting to inform the next step.

\section{Functional damage assessment}

After clamping, damage has accumulated in the different constituents. For the smooth muscle cells, this amount of damage can be calibrated and validated in a myograph, as explained in Sect. 4.1. The simulation starts from the same mesh as in step 1 of Sect. 4.2. This time, however, the initial conditions are specified for the solution-dependent variables taking into account the earlier loading history through the internal variables $\beta^{i}$. The material model is adapted, such that damage due to the energy accumulation of clamping is present, but no further damage is induced. Similar to step 1 of Sect. 4.2, the segment is closed to form a half cylinder in step 5, thus incorporating the circumferential residual stress. To reproduce the experimental situation, this time, no longitudinal stretch or internal pressure was added. Next, in step 6 , a rod is translated radially from inside the section, pulling it until it exerts a certain load, corresponding to the experimentally measured value after complete relaxation due to the addition of SNP. A friction coefficient of $\mu^{\text {rod }}=0.5$ is used between the rod and the outer arterial surface. Up to the end of step 6, no smooth muscle cell contribution is added in the material model. This is accomplished by multiplying 
the fractions $n_{\mathrm{III}}$ and $n_{\mathrm{IV}}$ with a switch function that is set to zero in steps 5 and 6.

After reaching the relaxed state, in the final step, the switch function is smoothly ramped to one, so that the smooth muscle cells reach the completely contracted state. Physiologically, this situation corresponds to the state after the addition of PE. In this step only, because of the time dependence of the evolution law for the relative sliding $u_{\mathrm{rs}}$, the time step of the implicit solution scheme is fixed to $\mathrm{dt}=10^{-5}$. Figure 5 gives a schematic overview of all seven steps of the simulation.

\section{Parameter selection}

Table 2 gives an overview of all parameters of the material model. The first set of parameters are related to the extracellular matrix with two embedded fibre families. For the rat abdominal aorta, the main direction of the collagen fibres $\alpha^{\mathrm{fib}}$ is set to $\pm 5^{\circ}$, that is, it is almost aligned with the circumferential direction, see O'Connell et al. (2008). The four remaining parameters are set to $\kappa=0.16(-), k_{1}=32.51$ $\mathrm{kPa}, k_{2}=3.05(-)$ and $c=23.63 \mathrm{kPa}$, using experimental data from extension-inflation tests as described in Famaey et al. (2012). Alternatively, a parameter set from human arteries can be found in Stålhand (2009).

The next set of parameters are the rate constants of the chemical model defining the fractions $n_{\text {III }}$ and $n_{\text {IV }}$ in equation (see Eq. 19). They are chosen according to Hai and Murphy (1988). These values led to the fractions of $n_{\mathrm{III}}=0.164$ and $n_{\mathrm{IV}}=0.547$, which were used as fixed input values into the mechanical model. Additional parameters are related to the mechanical model of the smooth muscle cell contribution. According to O'Connell et al. (2008), the smooth muscle cells of rat abdominal arteries are oriented circumferentially with $\alpha^{\mathrm{smc}}=0^{\circ}$. The parameter $\mu^{\mathrm{smc}}$ depending on the stiffness of the actin-myosin filament structure and the parameter $\kappa_{c}$ related to the driving force per crossbridge were both tuned to fit the experimental contraction measured in the myograph due to addition of PE for a previously undamaged segment, as described in Sect. 4.1. The viscous damping constant $\eta$ was set to $60 \mathrm{MPa}$ s, corresponding to the value used in Murtada et al. (2010).

To characterise damage progression appropriately, two parameters need to be calibrated for each constituent, plus two additional ones for the smooth muscle cells, totalling ten parameters. Since the myograph experiment only allows for damage quantification in the smooth muscle cells, with the current setup, no reasonable damage parameters can be defined for the extracellular matrix and the collagen fibres. Additional complementary experiments will be needed for this task, as discussed in Sect. 5. Accordingly, here, $\gamma^{\text {mat }}$ and $\gamma^{\text {fib }}$ were set to zero, such that $m^{\text {mat }}$ and $m^{\text {fib }}$ can take any arbitrary value. Secondly, the assumption was made that, during clamping, the smooth muscle cells were completely passive, and thus not contributing to the stiffness. Consequently, no damage could accumulate here, so that $\gamma_{\text {act }}^{\text {smc }}$ could also be set to zero, and $m_{\mathrm{act}}^{\text {smc }}$ to an arbitrary value. The two remaining parameters $\gamma_{\mathrm{pas}}^{\mathrm{smc}}$ and $m_{\mathrm{pas}}^{\mathrm{smc}}$ were then calibrated using the experimental data. For a systematic approach to calibrate damage material parameters in a heterogeneous setting, the reader is referred to Mahnken and Kuhl (1999).

\subsection{Results}

The top image in Fig. 6a shows the maximum principal stress in an arterial segment in the systolic physiological state. This state defines the damage threshold above which damage is initiated. In the lower image of Fig. 6a, the maximum principal stress is shown for the same arterial segment when clamped up to a clamping force of $5 \mathrm{mN}$. Figure $6 \mathrm{~b}$ shows the same set of images, this time displaying the elastic strain energy in the matrix material, $\widehat{\Psi}^{\text {mat }}$, that is, the driving force for both isotropic matrix damage $d^{\text {mat }}$ and passive smooth muscle cell damage $d_{\mathrm{pas}}^{\mathrm{smc}}$. As shown in the lower image of Fig. 6c, the clamping has induced an inhomogeneous damage pattern to the smooth muscle cells. Even when the segment returns to its reference state (top image in Fig. 6c), this damage is irreversible and remains.

Figure 7 shows snapshots of the myograph experiment, with the colour code depicting the maximum principal stress. The left graph of Fig. 8 shows the force measured in the rods of the myograph as a function of time, for a previously undamaged segment, solid line, and for a segment that was previously clamped at $5 \mathrm{mN}$, dashed. The letters along the curve correspond to the stages shown in Fig. 7. The first section of the graph corresponds to step 6 of the simulation, that is, the pulling of the rod to the passive state. After $2 \mathrm{~s}$, the smooth muscle cells are activated, corresponding to step 7.

The right graph of Fig. 8 shows the force measured in the rod for a segment that was previously clamped with the device described in Sect. 4.1 to a level of $5 \mathrm{mN}$, normalised to the width of the numerical model, and for a segment that was undamaged. The force in the rod was also normalised to the width of the numerical model. Again, in the first section of the graph, the rod is gradually pulled to reach the passive preload state. At the point indicated with the arrow, PE is added to the Krebs solution, triggering the activation of the smooth muscle cells. Note that the time scales in the two graphs do not agree. To calibrate the model appropriately, an additional time parameter would have to be included into the model. Here, however, we were only interested in the end result of the curve, rather than in calibrating the model to real physical times. 


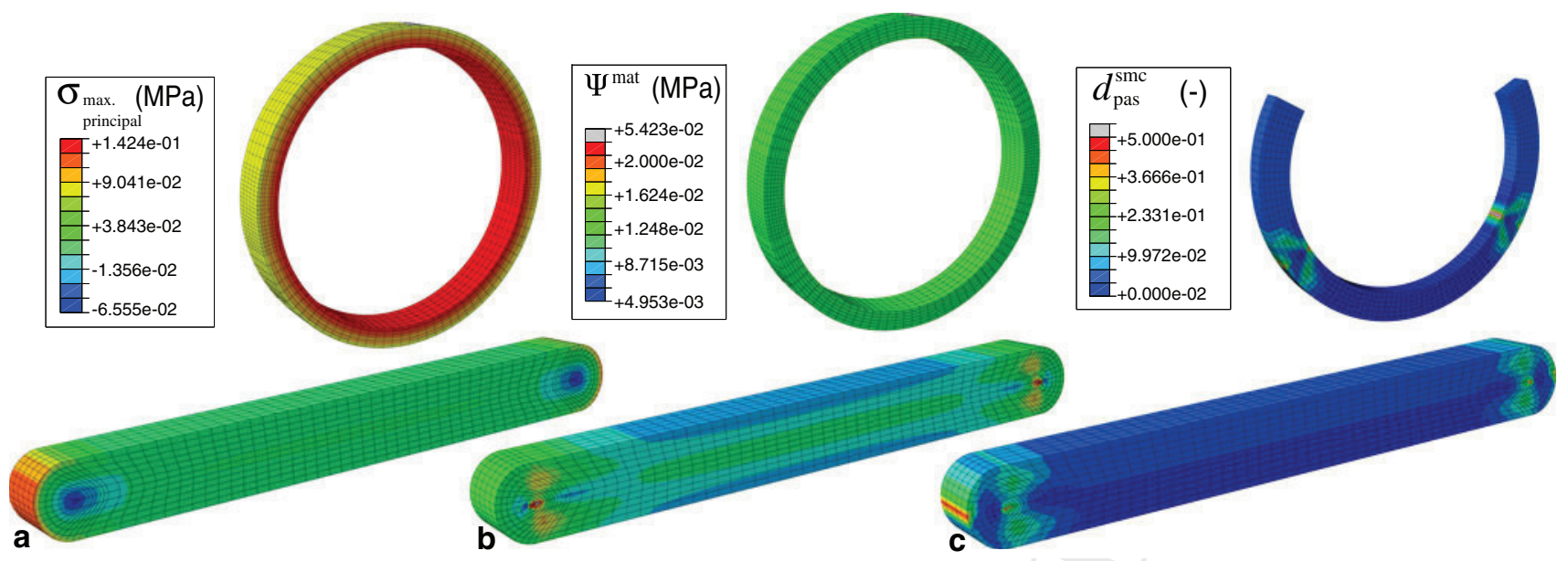

Fig. 6 a Maximum principal stress in an arterial segment in systolic physiological state (top image), and when clamped up to a clamping force of $5 \mathrm{mN}$ (lower image). b Strain energy in the same two segments as in (a). c Damage variable $d_{\mathrm{pas}}^{\mathrm{smc}}$ in the same two segments as in (a),

when clamped up to a clamping force of $5 \mathrm{mN}$ (lower image). This damage remains, even when the segment returns to its reference state (top image)
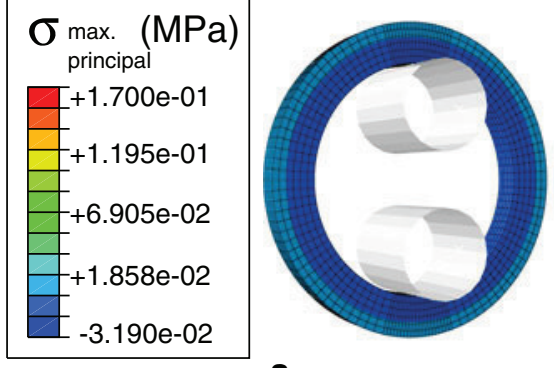

a

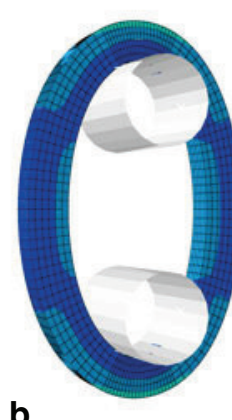

b

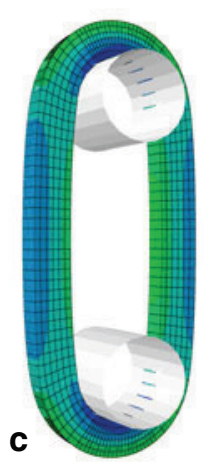

ulation). In stages $\mathrm{d}$, e and $\mathrm{f}$, the rods remain in position and the smooth muscle cells are activated (step 7 of the numerical simulation)
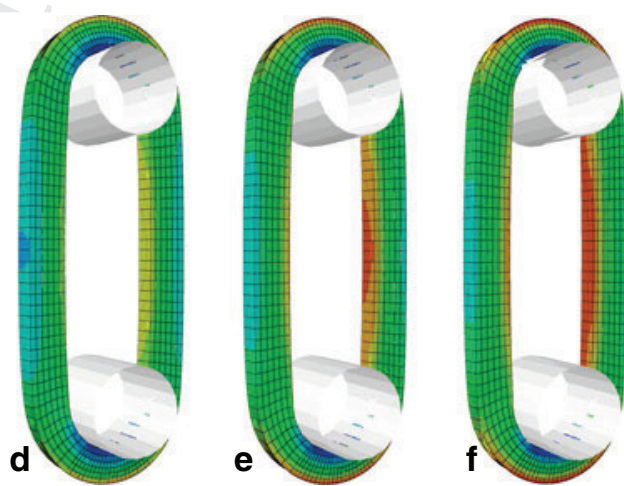

Fifferent stages of the myograph experiment, with the colc code depicting the maximum principal stress. In stages $\mathrm{a}, \mathrm{b}$ and $\mathrm{c}$, the rods are being pulled to the preload force (step 6 of the numerical sim-

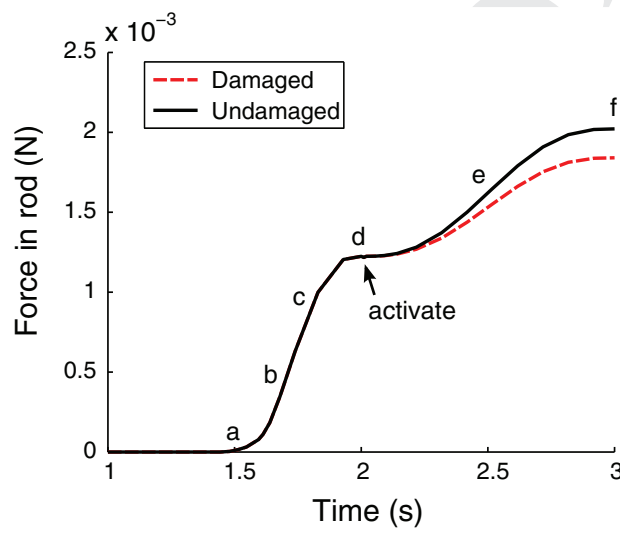

Fig. 8 The left graph shows the force measured in the rod of the myograph as a function of time, for a previously undamaged segment (solid line) and for a segment that was previously clamped at $5 \mathrm{mN}$ (dashed). The letters along the curve correspond to the snapshots shown in Fig.

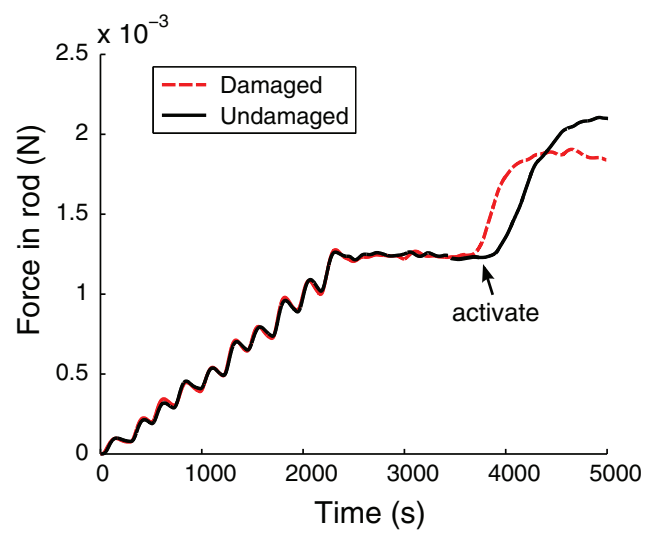

7. The right graph shows the force measured in the rod during an experiment, for a segment that was previously clamped with the device described in Sect. 4.1 to a level of $5 \mathrm{mN}$ and for a segment that was undamaged, both normalised to the width of the numerical model 


\section{Discussion}

In this article, a three-constituent damage constitutive model was proposed to simulate the damage process in arterial tissue. After testing the model in a homogeneous model problem under cyclic uniaxial tension and compression, it was used in a finite element simulation for the clamping of an artery and the subsequent damage evaluation in a myograph. The model enables the analysis of the inhomogeneous damage profile in the artery due to loading, quantitatively showing which constituents and which sections are overloaded, compared with the physiological state. In response to overload, driven by the free energy, anisotropic damage develops in the smooth muscle cells. The three-constituent damage model and numerical simulation provide a useful tool to explore safe loading of arterial tissue. Being able to reliably predict loading regimes which initiate tissue damage is important in view of robotic surgery, which lacks the natural feedback of human touch, by which the experienced surgeon today guarantees safe tissue loading.

The material model described in Sect. 2 introduces a large set of parameters, which need to be experimentally defined for each tissue type. Extensive experimental data from a range of different experiments is required to correctly calibrate all parameters. Section 4.2 comments on the rationale behind the parameter selection for this study. The goal of this study was to demonstrate the feasibility of the proposed model and to illustrate a conceptual methodology for the damage characterisation in smooth muscle cells. Accordingly, less emphasis was placed on the exact parameter identification for the other model parameters. As explained in Sect. 2.2, four damage processes can be captured by the model, one for each constituent. Each damage process is assumed to be driven by the individual free energy of that constituent. For smooth muscle cells, passive damage is also affected by the energy in the matrix constituent. Here, we focus in particular on this last passive part of damage, assuming that smooth muscle cells are inactive during the real clamping process. The damage parameters were chosen to correspond to the results of an ex vivo experiment. In the future, further experiments will be performed with different clamping force levels to calibrate the model for a wider loading range. To enable numerical comparison with higher clamping force levels, it might become relevant to remesh the clamped segment to avoid excessive element distortion. However, remeshing would require the mapping of the solution, both from the node points and from the integration points, onto the new mesh, a feature currently still lacking for anisotropic materials in Abaqus 6.10.

In order to accurately identify the damage parameters for the different constituents, different, ideally orthogonal, experiments are required that enable the extraction of this specific information. Damage in the collagen fibres under tension can possibly be studied using microscopic images of the tissue at different stages in the stretching process and assessing the images for collagen rupture. In fact, the extension-inflation tests that were used here to calibrate the undamaged baseline parameters of the Holzapfel model most probably already induced damage to both collagen fibres and matrix in the higher pressure regimes. Damage in the collagen fibres and matrix should therefore ideally be calibrated simultaneously, possibly through extension-inflation tests. Damage to the smooth muscle cells is assumed to depend on both damage of the passive extracellular matrix and damage of the active smooth muscle cells themselves. Damage in the passive regime has been observed and characterised experimentally in Famaey et al. (2010) and calibrated in this manuscript using these data. It results in a reduced activation capability, which will only become apparent upon activation. Damage in the active regime is caused by excessive tension in the direction of the contractile unit, which might cause ruptures in the myosin cross-bridges or rupture of the actin and myosin filaments. It is included here merely theoretically for the sake of completeness, but has not been calibrated yet. We are currently in the process of further investigating these phenomena to characterise the mechanisms underlying active damage.

Note also that in the finite element model, the artery was modelled as a single homogeneous layer, even though the wall consists of two solid mechanically relevant layers, that is, the media and the adventitia. However, in the case of a rat abdominal artery, the complete wall thickness is only approximately $0.14 \mathrm{~mm}$ thick, and in contrast to human tissue, it is impossible to separate these two layers from each other. Therefore, the most accurate approach was to model the wall as a single layer. The assumption was also made that damage initiates once the energy level exceeds that of the energy level at systolic blood pressure. This was motivated by the fact that the morphology and properties of the arterial wall change due to chronic hypertension (Matsumoto and Hayashi 1994), but whether this actually justifies this assumption for acute damage scenarios should still be experimentally validated.

Although the three-constituent damage model already captures a number of typical features of cardiovascular tissue, some characteristic aspects are still not included, and a few limitations remain. When qualitatively comparing the simulated homogeneous cyclic tension test described in Sect. 3.2 to the experimental results of a uniaxial tensile test on a sheep carotid artery, shown in Fig. 9, several features, for example, tissue nonlinearity and discontinuous softening are accurately captured. However, in the tensile test on the sheep carotid artery, cycling up to a certain strain level was performed five times before the next strain level was reached, and clearly softening does continue in these cycles, even though the maximum energy level, the parameter $\beta$ in our model is not increased. This continuous damage behaviour 


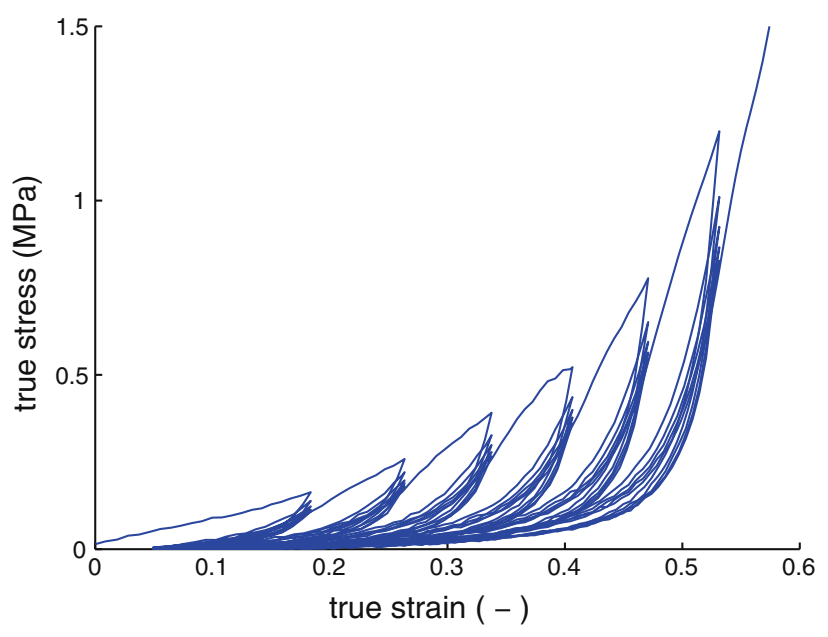

Fig. 9 Uniaxial tensile test on a circumferentially oriented strip of a sheep carotid artery. The test was performed on a tensile test bench (INSTRON 5567). Cyclic loading at gradually increasing levels of elongation was applied at a crosshead speed of $1 \mathrm{~mm} / \mathrm{s}$. The tests were performed with continuous recording of tensile force, with a $1 \mathrm{kN}$ load cell and gauge length, based on crosshead displacement, at a sampling frequency of $10 \mathrm{~Hz}$. Cycling up to a certain strain level was performed five times before the next strain level was reached, for six increasing levels of strain was not captured with the damage model used here. Moreover, the damage variables introduced in this model mainly capture acute effects, while chronic effects such as repair and/or remodelling have not been considered for the time being. These effects should be investigated, keeping in mind the trade-off between realism of the model and its usability. The correct identification of the material parameters obviously becomes more challenging as more effects are incorporated in the model.

The ultimate goal of this research project is to minimise tissue trauma during surgery, for which damage thresholds need to be identified. These thresholds should be defined in close collaboration with surgeons and biomedical researchers, experimentally assessing the level of damage due to loading and defining which damage levels are still acceptable, taking into account long-term effects of damage accumulation but also self healing. These critical damage levels can then be correlated to the internal damage variables $d$. Once the damage variable of a constituent has reached a certain level, the damage is set to be unacceptable, and robotic loading should be stopped automatically. Future research will therefore also be directed towards algorithm speed-up, for example, through parallelised implementation in the GPU with NVIDIA Compute Unified Device Architecture.

Predictive computational modelling of tolerable damage thresholds is clinically relevant in two ways: on the one hand, in the short term, the proposed model can be used as a simulation tool to optimise surgical tools, for example, to improve clamp design to minimise tissue damage. On the other hand, in the long term, the proposed model could enable the prediction of surgically induced damage evolution in real time. This would allow loading thresholds to be imposed on surgical instruments during an operation in a robotic teleoperation setting.

Acknowledgments This work was supported by a $\mathrm{PhD}$ grant from the Institute for the Promotion of Innovation through Science and Technology in Flanders (I.W.T.-Vlaanderen), a travel grant from the Research Foundation-Flanders, a travel grant from the Prof. R. Snoeys Foundation and a Fulbright scholarship.

\section{References}

Arruda EM, Boyce MC (1993) A three-dimensional constitutive model for the large stretch behavior of rubber elastic materials. J Mech Phys Solids 41(2):389-412

Balzani D, Schröder J, Gross D (2006) Simulation of discontinuous damage incorporating residual stresses in circumferentially overstretched atherosclerotic arteries. Acta Biomat 2(6):609-618

Balzani D, Schröder J, Gross D (2007) Numerical simulation of residual stresses in arterial walls. Comput Mater Sci 39:117-123

Balzani D, Böse D, Brads D, Erbel R, Klawonn A, Reinbach O, Schröder J (2011) Parallel simulation of patient-specific atherosclerotic arteries for the enhancement of intravascular ultrasound diagnosis. Eng Comp (submitted)

Barone GW, Conerly JM, Farley PC, Flanagan TL, Kron IL (1989) Assessing clamp-related vascular injuries by measurement of associated vascular dysfunction. Surgery 105(4):465-471

Böl M, Abilez OJ, Assar AN, Zarins CK, Kuhl E (2012) In vitro/in silico characterization of active and passive stresses in cardiac muscle. Int J Multiscale Comput Eng (in press)

Callera GE, Varanda WA, Bendhack LM (2000) Impaired relaxation to acetylcholine in $2 \mathrm{k}-1 \mathrm{c}$ hypertensive rat aortas involves changes in membrane hyperpolarization instead of an abnormal contribution of endothelial factors. Gen Pharmacol 34(6):379-389

Calvo B, Pena M, Martinez M, Doblaré M (2007) An uncoupled directional damage model for fibred biological soft tissues. Formulation and computational aspects. Int J Numer Methods Eng 69: 2036-2057

Dargazany R, Itskov M (2009) A network evolution model for the anisotropic mullins effect in carbon black filled rubbers. Int J Solids Struct 46(16):2967-2977

De S, Rosen J, Dagan A, Hannaford B, Swanson P, Sinanan M (2007) Assessment of tissue damage due to mechanical stresses. Int J Robot Res 26:1159-1171

Ehret A, Itskov M (2009) Modeling of anisotropic softening phenomena: application to soft biological tissues. Int J Plast 25:901-919

Famaey N, Vander Sloten J (2008) Soft tissue modelling for applications in virtual surgery and surgical robotics. Comput Methods Biomech Biomed Eng 11(4):351-366

Famaey N, Verbeken E, Vinckier S, Willaert B, Herijgers P, Vander Sloten J (2010) In vivo soft tissue damage assessment for applications in surgery. Med Eng Phys 32:437-443

Famaey N, Sommer G, Vander Sloten J, Holzapfel GA (2012) Experimental study and numerical analysis of arterial clamping. J Mech Behav Biomed Mater (accepted)

Fung YC (1970) Mathematical representation of the mechanical properties of the heart muscle. J Biomech 3(4):381-404

Gasser TC, Ogden RW, Holzapfel GA (2006) Hyperelastic modelling of arterial layers with distributed collagen fibre orientations. J R Soc Interface 3(6):15-35 
Gestrelius S, Borgström P (1986) A dynamic model of smooth muscle contraction. Biophys J 50(1):157-169

Gleason RL, Gray SP, Wilson E, Humphrey JD (2004) A multiaxial computer-controlled organ culture and biomechanical device for mouse carotid arteries. J Biomech Eng 126(6):787-795

Göktepe S, Kuhl E (2010) Electromechanics of the heart-a unified approach to the strongly coupled excitation-contraction problem. Comput Mech 45:227-243

Göktepe S, Acharya SNS, Wong J, Kuhl E (2011) Computational modeling of passive myocardium. Int J Numer Methods Biomed Eng 27:1-12

Gupta V, Reddy NP, Batur P (1997) Forces in laparoscopic surgical tools. Presence 6:218-228

Hai CM, Murphy RA (1988) Cross-bridge phosphorylation and regulation of latch state in smooth muscle. Am J Physiol 254(1 Pt 1):C99-106

Hill A (1938) The heat of shortening and the dynamic constants of muscle. Proc R Soc Lond B 126:136-195

Hokanson J, Yazdani S (1997) A constitutive model of the artery with damage. Mech Res Commun 24(2):151-159

Holzapfel GA, Ogden RW (2010a) Modelling the layer-specific threedimensional residual stresses in arteries, with an application to the human aorta. J R Soc Interface 7:787-799

Holzapfel GA, Ogden RW (2010b) Constitutive modeling of arteries. Proc R Soc Lond A 466:1551-1597

Holzapfel GA, Gasser TC, Ogden RW (2000) A new constitutive framework for arterial wall mechanics and a comparative study of material models. J Elast 61:1-48

Hsi C, Cuenoud H, Soller BR, Kim H, Favreau J, Salm TJV, Moran JM (2002) Experimental coronary artery occlusion: relevance to off-pump cardiac surgery. Asian Cardiovasc Thorac Ann 10(4):293-297

Itoh A, Krishnamurthy G, Swanson J, Ennis D, Bothe W, Kuhl E, Karlsson M, Davis L, Miller DC, Ingels NB (2009) Active stiffening of mitral valve leaflets in the beating heart. Am J Physiol Heart Circ Physiol 296:1766-1773

Kroon M (2010) A constitutive model for smooth muscle including active tone and passive viscoelastic behaviour. Math Med Biol 27(2):129-155

Kuhl E, Ramm E (1999) Simulation of strain localization with gradient enhanced damage models. Comput Mater Sci 16:176-185

Kuhl E, Maas R, Himpel G, Menzel A (2007) Computational modeling of arterial wall growth: Attempts towards patient specific simulations based on computer tomography. Biomech Model Mechanobiol 6:321-331

Kwoh YS, Hou J, Jonckheere EA, Hayall S (1988) A robot with improved absolute positioning accuracy for ct guided stereotactic brain surgery. IEEE Trans Biomed Eng 35:153-161

Mahnken R, Kuhl E (1999) Parameter identification of gradient enhanced damage models with the finite element method. Eur J Mech/A Solids 18:819-835

Manchio JV, Gu J, Romar L, Brown J, Gammie J, Pierson RN, Griffith B, Poston RS (2005) Disruption of graft endothelium correlates with early failure after off-pump coronary artery bypass surgery. Ann Thorac Surg 79(6):1991-1998

Matsumoto T, Hayashi K (1994) Mechanical and dimensional adaptation of rat aorta to hypertension. J Biomech Eng 116(3):278-283

Miehe C (1995) Discontinuous and continuous damage evolution in ogden-type large-strain elastic materials. Eur J Mech A/Solids 14:697-720
Mohr FW, Falk V, Diegeler A, Walther T, Gummert JF, Bucerius J, ${ }_{1027}$ Jacobs S, Autschbach R (2001) Computer-enhanced robotic car- 1028 diac surgery: experience in 148 patients. J Thorac Cardiovasc Surg 1029 121:842-853

Murtada S-I, Kroon M, Holzapfel GA (2010) A calcium-driven mechanochemical model for prediction of force generation in smooth muscle. Biomech Model Mechanobiol 9(6):749-762

O’Connell MK, Murthy S, Phan S, Xu C, Buchanan J, Spilker R, Dalman RL, Zarins CK, Denk W, Taylor CA (2008) The three-dimensional micro- and nanostructure of the aortic medial lamellar unit measured using $3 \mathrm{~d}$ confocal and electron microscopy imaging. Matrix Biol 27(3):171-181

Ogden RW, Roxburgh DG (1999) A pseudo-elastic model for the mullins effect in filled rubber. Proc R Soc A 455:2861-2877

Pena E, Alastrué V, Laborda A, Matrínez M, Doblaré M (2010) A constitutive formulation of vascular tissue mechanics including viscoelasticity and softening behaviour. J Biomech 43:984-989

Rausch MK, Dam A, Göktepe S, Abilez OJ, Kuhl E (2011) Computational modeling of growth: systemic and pulmonary hypertension in the heart. Biomech Model Mechanobiol 10(6):799-811

Rhodin JAG (1979) Architecture of the vessel wall. In: Berne RM (ed) Handbook of physiology, section 2, volume 2. Am. Physiol. Soc., Bethesda

Rodríguez JF, Cacho F, Bea JA, Doblaré M (2006) A stochastic-structurally based three dimensional finite-strain damage model for fibrous soft tissue. J Mech Phys Solids 54(4):864-886

Sacks MS, Sun W (2003) Multiaxial mechanical behavior of biological materials. Annu Rev Biomed Eng 5:251-284

Schmitz A, Böl M (2011) On a phenomenological model for active smooth muscle contraction. J Biomech 44:2090-2095

Simo J, Ju J (1987) Strain- and stress-based continuum damage models. Int J Solids Stuct 23:821-840

Stålhand J (2009) Determination of human arterial wall parameters from clinical data. Biomech Model Mechanobiol 8(2):141-148

Stålhand J, Klarbring A, Holzapfel GA (2008) Smooth muscle contraction: mechanochemical formulation for homogeneous finite strains. Prog Biophys Mol Biol 96:465-481

Stålhand J, Klarbring A, Holzapfel GA (2011) A mechanochemical $3 \mathrm{~d}$ continuum model for smooth muscle contraction under finite strains. J Theor Biol 268(1):120-130

Tsamis A, Bothe W, Kvitting JP, Swanson JC, Miller DC, Kuhl E (2011) Active contraction of cardiac muscle: in vivo characterization of mechanical activation sequences in the beating heart. $\mathrm{J}$ Mech Behav Biomed Mater 4:1167-1176

Vito RP, Dixon SA (2003) Blood vessel constitutive models-19952002. Annu Rev Biomed Eng 5:413-439

Volokh KY (2008) Prediction of arterial failure based on a microstructural bi-layer fiber matrix model with softening. J Biomech 41(2):447-453

Volokh KY (2011) Modeling failure of soft anisotropic materials with application to arteries. J Mech Behav Biomed Mater 4(8):15821594

Yang J, Clark JWJr, Bryan RM, Robertson C (2003) The myogenic response in isolated rat cerebrovascular arteries: smooth muscle cell model. Med Eng Phys 25(8):691-709

Zulliger MA, Rachev A, Stergiopulos N (2004) A constitutive formulation of arterial mechanics including vascular smooth muscle tone. Am J Physiol Heart Circ Physiol 287(3):H1335-H1343 1030 1031 1032 